\section{Recurrent fusion in pediatric AMKL}

James Downing and colleagues report the discovery of recurrent driver mutations in non-Down syndrome acute megakaryoblastic leukemia (non-DS-AMKL), including a CBFA2T3-GLIS2 fusion present in $27 \%$ of pediatric cases (Cancer Cell 22, 683-697, 2012). The authors sequenced the transcriptomes of 14 pediatric AMKL cases and found 7 with a balanced inversion on chromosome 16 resulting in an in-frame fusion of CBFA2T3 and GLIS2. Follow-up analyses in a larger set of pediatric and adult AMKL cases showed that this fusion was recurrent, restricted to pediatric cases and associated with unfavorable outcome. The authors also identified a NUP98-KDM5A fusion in $8 \%$ of pediatric cases, as well as recurrent mutations in GATA1 and JAK kinase genes. To examine the biological effects of the CBFA2T3-GLIS2 fusion, the authors transduced mouse hematopoietic cells with a retrovirus encoding CBFA2T3-GLIS2 and found that the fusion conferred increased capacity for self-renewal, with evidence for differentiation along the megakaryocytic lineage. They further showed that these effects on self-renewal were likely mediated by upregulation of bone morphogenetic protein (BMP) signaling. These findings provide insights into the biology of AMKL and identify CBFA2T3-GLIS2 as a new clinical marker with prognostic significance for pediatric patients with AMKL.

\section{TREM2 and Alzheimer's disease}

Two groups have independently identified a rare variant that is associated with susceptibility to late-onset Alzheimer's disease (LOAD), with an effect size similar to that of the $\varepsilon 4$ risk allele of apolipoprotein E. Researchers in the Alzheimer Genetic Analysis Group (N. Engl. J. Med., published online 14 November 2012; doi:10.1056/NEJMoa1211851) nominated TREM2 as a promising candidate because homozygous lossof-function TREM2 mutations cause Nasu-Hakola disease, a rare recessive, early-onset form of dementia with leukoencephalopathy and bone cysts. They generated exome sequence data sets and identified a TREM2 missense variant, $\mathrm{R} 47 \mathrm{H}$, which associated with LOAD in cohorts from North America and Europe. Separately, researchers at deCODE Genetics (N. Engl. J. Med., published online 14 November 2012; doi:10.1056/ NEJMoa1211103) identified the $\mathrm{R} 47 \mathrm{H}$ variant in an imputation-based genome-wide association study using the Icelandic population and replicated the association with LOAD in North American and European cohorts. TREM2 is an immune phagocytic receptor expressed in brain microglia. Assuming that the TREM2 risk variants impair TREM2 function, these studies suggest that reduced function of TREM 2 causes reduced phagocytic clearance of amyloid proteins or cellular debris and thus impairs a protective mechanism in the brain.

EN

\section{Migration from trees to graphs}

Joseph Pickrell and Jonathan Pritchard report a new statistical model for demographic inference from genome-wide allele frequency data sets (PLoS Genet, 8, e1002967, 2012). Implemented in the freely available software TreeMix (http://treemix.googlecode.com/), their approach involves building a population tree, finding populations that show poor fit to the tree model and modeling migration events to improve the fit within a graph-based model. They tested their method in simulations as well as on a human data set including genome-wide data from 53 modern and

Written by Orli Bahcall, Pamela Feliciano, Emily Niemitz \& Kyle Vogan
2 archaic populations. They inferred a maximum-likelihood tree from the human data set that recapitulates known population relationships and explains $98.8 \%$ of the variance in relatedness between populations. By sequentially adding the ten migration events that are most consistent with known events, they increased the explained variance to $99.8 \%$. The authors also applied their approach to a canine genome-wide data set of 82 dog breeds or wild canids. They found that a significant amount of gene flow occurred between breeds during dog domestication and again demonstrate the ability to increase the explained variance in relatedness between dog breeds by sequentially adding migration events. For both humans and dogs, the authors infer many migration events, including previously known and new relationships between populations.

$O B$

\section{MED12 in cancer drug resistance}

Targeted cancer drugs do not typically lead to long-term survival benefits owing to emergent drug resistance caused by secondary-site mutations in the targeted gene or mutations in genes downstream. René Bernards and colleagues report that suppression of MED12 confers drug resistance to multiple targeted cancer drugs in multiple cancer cell lines (Cell 151, 937-950, 2012). The authors conducted an in vitro RNA interference (RNAi) screen targeting 8,000 genes. They found that $M E D 12$ was the only gene whose suppression led to resistance to the ALK inhibitor crizotinib. They then performed an inverse screen with a short hairpin RNA (shRNA) library that covers all 518 human kinases to identify genes whose suppression would restore drug sensitivity. They found that suppression of TGFBR2 resensitized MED $12^{\mathrm{KD}}$ cells to crizotinib. Further experiments showed that treatment of multiple cancer cell lines with recombinant TGF- $\beta$ conferred resistance to multiple targeted cancer drugs, as well as the widely used chemotherapy drug cisplatin. Finally, the authors tested the combination of a TGF- $\beta$ R2 inhibitor with crizotinib in $M E D 12^{\mathrm{KD}}$ cells and observed a synergistic inhibitory effect. The authors suggest that the combination of TGF- $\beta$ R2 inhibitors with targeted tyrosine kinase inhibitors might be an effective therapy for tumors with elevated TGF- $\beta$ signaling.

\section{Autism candidate gene resequencing}

Although much effort has been made in sequencing autism exomes, it has been difficult to robustly establish ASD (autism spectrum disorder) candidate genes as bona fide genetic risk factors, as only single mutations are typically observed. Now, Evan Eichler, Jay Shendure and colleagues report ultra-low-cost ASD candidate gene resequencing of 44 genes in 2,446 ASD probands (Science, published online 15 November 2012; doi:10.1126/ science.1227764). This resequencing method uses a modified molecular inversion probe (MIP) strategy, such that reagent costs are less than $\$ 1$ per gene per sample. Applying this method to ASD probands from the Simons Simplex Collection (SSC), the authors identified 27 de novo mutations in 16 genes, with $59 \%$ of the mutations predicted to be truncating or disruptive of splicing. Six of the genes (CHD8, GRIN2B, DYRK1A, PTEN, $T B R 1$ and TBL1XR1) have statistically significant evidence of mutation burden; five of these are contained within the $\beta$-catenin/ chromatin-remodeling network. Altogether, approximately $1 \%$ $(24 / 2,573)$ of ASD probands had a mutation in 1 of these 6 genes. Because the SSC was established for families with simplex ASD and these probands typically possess higher cognitive functioning levels than other ASD cohorts, it is not known how generalizable the mutation burdens of these six genes will be in idiopathic autism. 